\title{
ON THE CONNECTED IDENTITY COMPONENT OF THE ADELLE-CLASS GROUP OF AN ALGEBRAIC TORUS
}

CARL S. WEISMAN

The structure of the connected component of the identity of the idèle-class group of a number field is given in Artin-Tate [1]. It seems worthwhile to investigate whether this result is the "trivial case" of a theorem in the arithmetic of algebraic tori, i.e. the case corresponding to the multiplicative group $G_{m}$ (as is true of the unit theorem and the finiteness of the class number). It turns out that the formalism of the theory of tori defined over number fields allows one to generalize Artin's proof line-by-line, obtaining the structure of the identity component of the adèle-class group of any such torus. I am grateful to Professor T. Ono for suggesting the problem to me.

I give now a summary of the definitions and results that I shall need (for all this, see Ono [3]). I denote by $Z, Q, R$, and $C$ the rings of respectively integers, rationals, real numbers, and complex numbers, and by $T$ an algebraic torus defined over a number field $k . \hat{T}$ is the group of rational characters of $T$. If the dimension of $T$ is $d$, then $\hat{T}$ is a free abelian group of rank $d[3, \S 1.1] . T$ is split by a finite extension $K$ of $k$, which I choose to be normal over $\boldsymbol{Q}$. Then the group $T_{K}$ of points of $T$ rational over $K$ is isomorphic to the product $\left(K^{*}\right)^{d}$ of $K^{*}$ with itself $d$ times. Every $\xi \in \hat{T}$ is a rational map defined over $K$; hence if $g$ denotes the galois group of $K$ over $k, \hat{T}$ has the structure of a $\mathfrak{g}$-module. The group $\operatorname{Hom}\left(\hat{T}, K^{*}\right)$ has the structure of a $\mathfrak{g}$ module, given by $f^{\sigma}(\xi)=\left(f\left(\xi^{-1}\right)\right)^{\sigma}$, and we have $\mathrm{g}$-isomorphisms

$$
T_{K} \stackrel{\sim}{\rightarrow} \operatorname{Hom}\left(\hat{T}, K^{*}\right), \quad T_{k} \stackrel{\sim}{\rightarrow} \operatorname{Hom}_{\mathfrak{g}}\left(\hat{T}, K^{*}\right),
$$

where the isomorphisms are both given by the evaluation map.

For a valuation $v$ of $k, T_{o_{v}}$ denotes the maximal compact subgroup of $T_{k_{v}}$. The adelization $T_{A_{k}}$ of $T$ is defined as the restricted product of the group $T_{k_{v}}$ with respect to the subgroups $T_{o_{v}} . T_{k}$ is imbedded in $T_{A_{k}}$ on the diagonal and as such is a discrete subgroup. My object of study is the group $C_{k}(T)=T_{A_{k}} / T_{k}$ of adèle classes of $T$. The subgroup $T_{A_{k}}\left(S_{\infty}\right)$ of $T_{A_{k}}$ is by definition $\tilde{U} \bar{U}$, where

$$
\tilde{U}=\prod_{v \mid \infty} T_{k_{v}} \times \prod_{v \text { finite }}\{1\}
$$

and

Received by the editors March 20, 1967. 


$$
\bar{U}=\prod_{v \mid \infty}\{1\} \times \prod_{\bullet \text { finite }} T_{0_{v}} .
$$

I write an element $\mathfrak{a} \in T_{\boldsymbol{A}_{\boldsymbol{k}}}\left(S_{\infty}\right)$ as $\tilde{\mathfrak{a}} \overline{\mathfrak{a}}$, where $\tilde{\mathfrak{a}} \in \tilde{U}$ and $\mathfrak{a} \in \bar{U}$. A rational character $\xi$ that is defined over $k$ induces a homomorphism $\xi: T_{\boldsymbol{A}_{k}} \rightarrow J_{k}$, where $J_{k}$ is the idèle group of $k . T_{\boldsymbol{A}_{k}}$ denotes the group

$$
\left\{\mathfrak{a} \in T_{A_{k}} \mid\|\xi(\mathfrak{a})\|=1 \text { for all } \xi \in \hat{T}_{k}\right\}
$$

where $\|\cdot\|$ denotes the idèle-module. We have that $T_{k} \subset T_{A_{k}}^{1}$ and that $T_{A_{k}}^{1} / T_{k}$ is compact [2, Theorem 2].

Define $T_{A k}^{1}\left(S_{\infty}\right)=T_{A_{k}}\left(S_{\infty}\right) \cap T_{A_{k}}^{1}$. The finitely generated group $T_{k} \cap T_{A_{k}}\left(S_{\infty}\right)$ is called the group of units of $T$.

Associated to $T$, we have the torus $T_{0}=R_{k / Q}(T)$, the restriction of $T$ from $k$ to $\boldsymbol{Q}$. It is a torus defined over $\boldsymbol{Q}$, of dimension $d n$, where $d$ is the dimension of $T$ and $n=[k: Q]$. We have the following canonical isomorphisms:

$$
\begin{aligned}
& \left(T_{0}\right)_{8} \cong T_{k}, \\
& \left(T_{0}\right) z_{v_{0}} \cong \prod_{\boldsymbol{v} \mid v_{0}} T_{\mathrm{v}_{\boldsymbol{v}}}, \quad\left(T_{0}\right)_{\mathcal{} Q} \cong T_{A_{k}}, \\
& \left(T_{0}\right)_{A_{Q}}(\infty) \cong T_{A_{k}}\left(S_{\infty}\right), \quad\left(T_{0}\right)_{A Q}^{1} \cong T_{A_{k}}^{1} .
\end{aligned}
$$

For the purposes of this paper, I regard these various groups as being identified. Moreover, I identify the unit group of $T_{0}$ with that of $T$, and $\left(T_{0}\right)_{R}$ with $\tilde{U}$.

Let $\rho_{R}$ be the rank of $\left(\hat{T}_{0}\right)_{R}$. Then $\left(T_{0}\right)_{R} /\left(T_{0}\right)_{0_{\infty}} \cong R^{\rho_{R}}[3, \S 2.1]$, where $\left(T_{0}\right)_{0_{\infty}}$ denotes the maximal compact subgroup of $\left(T_{0}\right)_{R} \cdot\left(T_{0}\right)_{R}$ is a Lie group of dimension $d n$. The maximal compact subgroup of the identity component of $\left(T_{0}\right)_{R}$ is a compact connected abelian Lie group. Hence it is a product of circle groups, the number being $d n-\rho_{R}$. I write it as the image of $R^{d n-\rho_{R}}$ via a homomorphism $\Phi$ whose kernel is $Z^{d n-\rho_{R}}$.

If $\rho_{Q}$ is the rank of $\left(\hat{T}_{0}\right)_{Q}$, then the unit group of $T_{0}$ has rank $\rho=\rho_{R}-\rho_{Q}\left[2\right.$, Theorem 4]. The identity component of $\left(T_{0}\right)_{R}$ has finite index in $\left(T_{0}\right)_{R}$ [G. D. Mostow, On the fundamental group of a homogeneous space, Ann. of Math. 66 (1957), 249-255], so there exists a linearly independent set $\epsilon_{1}, \cdots, \epsilon_{\rho}$ of torsion-free units of $T_{0}$ (and hence of $T$ ) such that each $\tilde{\epsilon}_{j}$ lies in the identity component of $\tilde{U}\left(=\left(T_{0}\right)_{R}\right)$.

The collection of open subgroups of finite index in $\bar{U}$ forms a fundamental system of neighborhoods of the neutral element. Hence, as in [1], $\tilde{\epsilon}_{j}^{x}$ is a well-defined element of $\bar{U}$, where $x$ is an element of the 
completion $\overline{\boldsymbol{Z}}$ of $\boldsymbol{Z}$ with respect to the topology of subgroups of finite index.

LEMMA 1. If $x_{1}, \cdots, x_{\rho}$ are elements of $\bar{Z}$ such that

$$
\tilde{\epsilon}_{1}^{x_{1}} \tilde{\epsilon}_{2} \ldots \tilde{\epsilon}_{\rho}^{x_{\rho}}=1,
$$

then $x_{1}=x_{2}=\cdots=x_{p}=0$.

Proof. Since $\left(T_{0}\right)_{K}$ is isomorphic to $\left(K^{*}\right)^{n d}, \epsilon_{1}, \cdots, \epsilon_{\rho}$ are independent elements of the $n d$ th power of the group of units of $K$ whose archimedean parts lie in the connected component of the archimedean part of $\left(J_{K}\right)^{n d}$. Hence the result follows by an evident extension of Lemma 1 of [1], applied to $K$.

Choose a basis $\xi_{1}, \cdots, \xi_{d n}$ of $T_{0}$ over $\boldsymbol{Z}$ such that $\xi_{1}, \cdots, \xi_{\rho Q}$ that $x_{j} \equiv n_{j}(\bmod 2 M \nu N)$. Then $\bar{\epsilon}_{j}^{n_{j}-x_{j}}=\bar{a}_{j}$, where for each finite $p, \bar{a}_{j p}$ is a $2 M \nu N$-th power in $\left(T_{0}\right)_{z_{p}}$. If $\epsilon=\epsilon_{1}^{n_{1}} \cdots \epsilon^{n_{p}}$, then

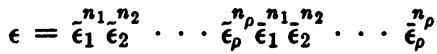

$$
\begin{aligned}
& =\tilde{\epsilon}_{1}^{n_{1}} \cdots \tilde{\epsilon}_{\rho}^{n_{\rho} \bar{\epsilon}_{1} \bar{\epsilon}_{1} \bar{\epsilon}_{2}} \cdots \bar{\epsilon}_{\rho}^{x_{\rho}} \bar{a}_{1} \bar{a}_{1} \cdots \bar{a}_{\rho} \\
& =\tilde{\epsilon}_{1}^{n_{1}} \cdots \tilde{\epsilon}_{\rho}^{n_{\rho}} \bar{a}_{1} \cdots \bar{a}_{\rho} \text {. }
\end{aligned}
$$

If we regard $\epsilon$ as being contained in one of the factors of $\left(T_{0}\right)^{m}$, then for any finite $p, \alpha(\epsilon)$ is a $2 M \nu N$ th power in $\left(\prod_{\mu}\left(R_{k_{\mu} / Q}\left(G_{m}\right)\right)^{m_{\mu}}\right)_{Q_{p}}$. By an evident extension of Theorem 1 of [1] (using the fact that $\left.R_{k_{\mu} / Q}\left(G_{m}\right)_{Q} \simeq k_{\mu}^{*}\right)$, this implies that $\alpha(\epsilon)=\eta^{M \nu N}$, for some

$$
\eta \in\left(\prod_{\mu}\left(R_{k_{\mu} / \theta}\left(G_{m}\right)\right)^{m_{\mu}}\right)_{Q}
$$

So $\beta(\eta)^{M \nu N}=\beta(\alpha(\epsilon))=\epsilon^{\nu}[3, \S 1.3] . \beta(\eta)$ is a unit. $\beta(\eta)^{M}$ lies in the group generated by $\epsilon_{1}, \cdots, \epsilon_{\rho}$. Therefore $N \mid n_{j}, j=1, \cdots, \rho$. Hence each $x_{j}$ lies in the closure of $N Z$. But $N$ was arbitrary, so each $x_{j}=0$.

Choose a basis $\xi_{1}, \cdots, \xi_{d n}$ of $\hat{T}_{0}$ over $\boldsymbol{Z}$ such that $\xi_{1}, \cdots, \xi_{\rho Q}$ form a basis for $\left(\hat{T}_{0}\right)_{Q}$ and $\xi_{1}, \cdots, \xi_{p_{R}}$ form a basis for $\left(\hat{T}_{0}\right)_{R}[3, \S 3.8]$. For $i \leqq \rho_{R}$ and $j \leqq \rho, \xi_{i}\left(\epsilon_{j}\right)>0$, since $\tilde{\epsilon}_{j}$ lies in the identity component of $\tilde{U}$. The following is proved in [2, Theorem 4]:

LEMMA 2. If $s_{1}, \cdots, s_{p}$ are real numbers such that

$$
\sum_{j=1}^{p} s_{j} \log \xi_{i}\left(\epsilon_{j}\right)=0, \quad i=1, \cdots, \rho_{R},
$$

then $s_{1}=s_{2}=\cdots=s_{p}=0$.

If $s \in R$ consider the homomorphism $u: \hat{T}_{0} \rightarrow R^{*}$ defined as follows: if $\xi=\prod_{i=1}^{d n} m_{i} \xi_{i} \in \hat{T}_{0}$, then 


$$
u(\xi)=\prod_{i=1}^{\rho R} \xi_{i}\left(\epsilon_{j}\right)^{s m_{i}}
$$

This defines an element of $\left(T_{0}\right)_{C}$, which in fact must lie in $\left(T_{0}\right)_{R}$, since $u$ is fixed under complex conjugation. I denote this element $\tilde{\epsilon}_{j}^{s}$.

Let $V=R \times \overline{\mathbf{Z}}$. For each $j \leqq \rho$ we have an evidently continuous homomorphism $\lambda \rightarrow \epsilon_{j}^{\lambda}$ taking $V \rightarrow T_{A_{k}}\left(S_{\infty}\right)$, defined by $\epsilon_{j}^{\lambda}=\tilde{\epsilon}_{j}^{s} \bar{\epsilon}_{j}^{x}$, where $\lambda=(s, x)$. Let $i \leqq \rho_{Q}$. Then by definition, $\xi_{i}\left(\tilde{\epsilon}_{j}^{\delta}\right)=\xi_{i}\left(\tilde{\epsilon}_{j}\right)^{s}$. Now $\xi_{i}\left(\epsilon_{j}\right)$ $= \pm 1$, since $\epsilon_{j}$ is a unit and $\xi_{i} \in\left(\hat{T}_{0}\right)_{Q}$. But $\xi_{i}\left(\epsilon_{j}\right)>0$, so $\xi_{i}\left(\tilde{\epsilon}_{j}\right)=1$. Let $n_{\alpha}$ be a net of ordinary integers converging to $x$. Then

$$
\xi_{i}\left(\bar{\epsilon}_{j}^{x}\right)=\lim \xi_{i}\left(\bar{\epsilon}_{j}^{n \alpha}\right)=\lim \xi_{i}\left(\bar{\epsilon}_{j}\right)^{n \alpha}=1 .
$$

Hence $\epsilon_{j}^{\lambda} \in T_{A k}^{1}\left(S_{\infty}\right)$.

LEMmA 3. A necessary and sufficient condition that $\epsilon_{1}^{\lambda_{1}} \cdots \epsilon_{\rho}^{\lambda \rho} \Phi(t)$ lie in $T_{k}$ is that $t \in \boldsymbol{Z}^{\boldsymbol{d}_{n-\rho}} \boldsymbol{R}$ and each $\lambda_{j} \in \boldsymbol{Z}$, where $\boldsymbol{Z}$ is regarded as imbedded in $R \times \bar{Z}$ on the diagonal.

Proof. The sufficiency is evident, so let

$$
\stackrel{\lambda_{1}}{\epsilon_{1}} \ldots{\stackrel{i}{\epsilon_{\rho}}}^{\lambda_{\rho}} \Phi(t)=\alpha \in T_{k} .
$$

Then $\alpha$ is a unit, so for some natural number $M$,

$$
\alpha^{M}={ }_{\epsilon_{1}}^{\mu_{1}} \cdots \epsilon_{\rho}^{\mu_{\rho}},
$$

where the $\mu_{j}$ are ordinary integers. But at the finite primes we are given that

$$
\bar{\alpha}=\bar{\epsilon}_{1}^{x} \ldots \bar{\epsilon}_{\rho}^{x_{\rho}} .
$$

Consequently,

$$
\bar{\epsilon}_{1}^{M x_{1}-\mu 1} \cdots \bar{\epsilon}_{\rho}^{M x_{\rho}-\mu_{\rho}}=1 .
$$

By Lemma 1, each $M x_{j}-\mu_{j}=0$, so each $x_{j}$ is an ordinary integer and $\alpha$ differs from $\epsilon_{1}^{x_{1}} \cdots \epsilon_{\rho}^{x_{\rho}}$ by a unit of finite order. Thus

$$
\tilde{\epsilon}_{1}^{s_{1}-x_{1}} \cdots \tilde{\epsilon}_{\rho}^{s_{\rho}-x_{\rho}} \Phi(t)=u,
$$

where $u$ is a unit of finite order. The image of $\Phi$ being compact and connected, $\xi_{i}(\Phi(t))=1$ for each $i \leqq \rho_{R}$. Since $u$ has finite order, it lies in the maximal compact subgroup of $\left(T_{0}\right)_{R}$. On the other hand, it is given as equal to an element in the identity component of $\left(T_{0}\right)_{R}$, so $\xi_{i}(u)=1$ for $i \leqq \rho_{R}$. Hence

$$
\sum_{j=1}^{\rho}\left(s_{j}-x_{j}\right) \log \xi_{i}\left(\epsilon_{j}\right)=0 .
$$


By Lemma 2, $s_{j}=x_{j}$ and so $\Phi(t)=u$. The component of $\Phi(t)$ at any finite prime is 1 ; since $u$ is a unit, this implies $\Phi(t)=1$, i.e. $t \in Z^{d n-p} R$.

By Lemma 3, we have a one-to-one continuous homomorphism of

$$
\begin{gathered}
V / Z \times \cdots \times V / Z \times R / Z \times \cdots \times R / Z \\
(\rho \text { times }) \\
\left(d n-\rho_{R} \text { times }\right)
\end{gathered}
$$

into $C_{\mathbf{k}}^{\mathbf{1}}(T)=T_{A k}^{1} / T_{k}$.

Lemma 4. The image of this map is the identity component of $C_{\boldsymbol{k}}^{1}(T)$.

Proof. The identity component of $C_{\boldsymbol{k}}^{1}(T)$, being a compact connected abelian group, is divisible. The domain of the homomorphism is connected [1]; hence it will be enough to show that every divisible element of $C_{\mathbf{k}}^{1}(T)$ lies in the image. Let

$$
h=\left[T_{A_{k}}^{1}: T_{k} \cdot T_{A_{k}}^{1}\left(S_{\infty}\right)\right]
$$

be the class number of $T$. (The index is finite since $T_{A k}^{1} / T_{k}$ is compact and $T_{A_{k}}^{1}\left(S_{\infty}\right)$ is open in $T_{A_{k}}^{1}$. $)$ Let $M$ be the index of the identity component of $\left(T_{0}\right)_{R}$ in $\left(T_{0}\right)_{R}$. For a divisible element $a \in C_{k}^{1}(T)$, we may write $a=b^{M h N}$ for any $N$. A representative of $b^{h}$ may be chosen in $T_{A_{k}}^{1}\left(S_{\infty}\right) . b^{M h}$ has a representative $\mathfrak{c}=\tilde{\mathfrak{c}} \overline{\mathfrak{c}}$ such that $\tilde{\mathfrak{c}}$ lies in the identity component of $\tilde{U}$. Then $\mathrm{c}^{N}$ is a representative of $a$. As $N$ becomes highly divisible, $\bar{c}^{N}$ approaches the identity. Since $V / \boldsymbol{Z} \times \cdots \times \boldsymbol{R} / \boldsymbol{Z}$ is compact, it will be enough to show that each $\tilde{\mathfrak{c}}^{N}$ lies in the image.

So let $\tilde{\mathfrak{a}}$ be any element of the identity component of $\tilde{U}$. Solve for $s_{1}, \cdots, s_{\rho}$ the equations

$$
\sum_{j=1}^{\rho} s_{j} \log \xi_{i}\left(\epsilon_{j}\right)=\log \xi_{i}(\tilde{\mathfrak{a}}), \quad \rho_{Q}+1 \leqq i \leqq \rho_{R} .
$$

Then for $\rho_{Q}+1 \leqq i \leqq \rho_{R}$,

$$
\xi_{i}\left(\tilde{\tilde{a}} \tilde{\epsilon}_{1}^{-s_{1}} \ldots \tilde{\epsilon}_{p}^{-s_{p}}\right)=1 .
$$

Since $\mathfrak{a} \in T_{\boldsymbol{A}_{\boldsymbol{k}}}^{\mathbf{1}}$,

$$
\xi_{i}\left(\tilde{\mathfrak{a}} \tilde{\epsilon}_{1}^{-s_{1}} \ldots \tilde{\epsilon}_{\rho}^{-s_{p}}\right)=1
$$

for $i \leqq \rho_{Q}$ as well. Since $\tilde{\mathfrak{a}} \tilde{\epsilon}_{1}^{-s_{1}} \cdots \tilde{\boldsymbol{\epsilon}}^{-s_{\rho}}$ is annihilated by every $\xi \in\left(T_{0}\right)_{R}$, it lies in the maximal compact subgroup of the identity component of $\left(T_{0}\right)_{R}$, i.e. in the image of $\Phi$.

To determine the structure of the identity component of $C_{k}(T)$ $=T_{A_{k}} / T_{k}$, let $j \leqq \rho_{Q}$ and let $u_{j}$ be the homomorphism $\hat{T}_{0} \rightarrow C^{*}$ defined by $u_{j}\left(\xi_{j}\right)=e$ and $u_{j}\left(\xi_{i}\right)=1$ for $i \neq j$. Then $u_{j}$ is in a natural way an 
element of the identity component of $\tilde{U}$. The group

$$
\left\{u_{1}^{s_{1}} u_{2}^{s_{2}} \cdots u_{p_{Q} \rho_{Q}} \mid s_{1}, \cdots, s_{\rho_{Q}} \in R\right\}
$$

is isomorphic to $R^{\rho} \theta$ and is mapped one-to-one modulo $T_{k}$. Its image is clearly a complementary subgroup to the identity component of $C_{\boldsymbol{k}}^{\mathbf{1}}(T)$. Hence we have the

THEOREM. The identity component of $C_{k}(T)$ is the product of $\rho_{Q}$ real lines, $d n-\rho_{R}$ circles, and $\rho$ rational solenoids.

\section{BiBLIOGRAPHY}

1. E. Artin and J. Tate, Class field theory, Chapter 9, Harvard University, 1961.

2. T. Ono, On some arithmetic properties of linear algebraic groups, Ann. of Math. 70 (1959), 266-290.

3. - Arithmetic of algebraic tori, Ann. of Math. 74 (1961), 101-139.

University of Pennsylvania 